\title{
Spontaneous Anterior Neck Hematoma Associated With Rivaroxaban Leading to Primary Hyperparathyroidism and Hypercalcemia
}

\author{
Leonid Shamban ${ }^{\mathrm{a}, \mathrm{c}}$, Brijesh Patel ${ }^{\mathrm{a}}$, Adam Forman ${ }^{\mathrm{a}}$, Naseer Ahmad
}

\begin{abstract}
New anticoagulation agents are now widely used for many indications. Post-marketing surveillance is intended to discover rare medication events that did not occur in clinical trials. The literature cites few reported cases of spontaneous neck hemorrhage while on anticoagulation. We report a case of an 86-year-old female with chronic atrial fibrillation who presented with spontaneous neck hematoma in the area of the parathyroid glands resulting in hyperparathyroidism and hypercalcemia while taking rivaroxaban. The hematoma spanned the anterior aspects of the neck and was confirmed via CT. The patient's anticoagulation therapy was discontinued and required intubation for airway protection. The parathyroid hormone (PTH) peaked to $176 \mathrm{mg} / \mathrm{dL}$ (upper limit of normal $65 \mathrm{pg} / \mathrm{mL}$ ) followed by close to normalization of PTH and corrected calcium after appropriate treatment and stabilization of the hematoma, which did not require surgical evacuation.
\end{abstract}

Keywords: Neck hematoma; Hyperparathyroidism; Hypercalcemia; Rivaroxaban

\section{Introduction}

A neck hematoma commonly occurs after procedures in head, neck and cervical regions, and trauma. However, without a history of surgery or trauma a spontaneous neck hematoma is a rare event. Typical etiologies may occur from dissection/aneurysm of vasculature structures, infection and coagulopathy [1]. The more unusual causes of spontaneous hematoma include

Manuscript accepted for publication June 09, 2014

aDepartment of Internal Medicine, Providence Hospital and Medical Center, 16001 W Nine Mile Road, Southfield, MI 48075, USA

bDepartment of Endocrinology, Providence Hospital and Medical Center, 16001 W Nine Mile Road, Southfield, MI 48075, USA

${ }^{\mathrm{c} C o r r e s p o n d i n g ~ A u t h o r: ~ L e o n i d ~ S h a m b a n, ~ D e p a r t m e n t ~ o f ~ I n t e r n a l ~ M e d i c i n e, ~}$ Providence Hospital and Medical Center, 16001 W Nine Mile Road, Southfield, MI 48075, USA. Email: 1shamban@gmail.com

doi: http://dx.doi.org/10.14740/jmc1825w ruptured thyroid or parathyroid adenoma. Most often, parathyroid adenoma investigation occurs with hypercalcemia and its clinical manifestations. In the literature there have been a few cases described of hypercalcemia presenting after a spontaneous rupture of the extra-capsular parathyroid adenoma leading to a cervico-mediastinal hematoma. The severity can range from self-limited bleeding to compromise of surrounding neuro-vasculatures and the airway [2]. Further for patients on anticoagulants the odds of severe bleeding significantly increase. A case report of spontaneous retropharyngeal hematoma with concomitant use of warfarin has been reported [3]. At present with many new oral anticoagulation agents widely used for various indications, post-marketing surveillance has become vital to discover rare medication events that were not discovered in clinical trials. To our knowledge there have been no reports of a spontaneous neck hematoma in a patient who has been taking rivaroxaban therapy from suspected rupture of parathyroid adenoma.

\section{Case Report}

An 86-year-old African American female with a history of chronic atrial fibrillation (Afib), diastolic heart failure, hypertension, chronic obstructive pulmonary disease oxygen-dependent and history of multiple deep vein thrombosis (DVT) and pulmonary embolism (PE) presented to the hospital secondary to acute progressive neck swelling and dysphagia. Two weeks prior to admission the patient was switched to daily rivaroxaban from daily warfarin therapy in order to increase her medication adherence. The patient was on warfarin for many years, given her CHADS2-VASc score of 5 along with her history of thromboembolic disease.

In the emergency department (ED) the patient's vital signs were blood pressure of $137 / 68 \mathrm{~mm} \mathrm{Hg}$, pulse of 87 beats per $\mathrm{min}$, respiratory rate of 20 breaths per min, temperature of 97.6 ${ }^{\circ} \mathrm{F}$ and pulse oximetry of $100 \%$ on $2 \mathrm{~L}$ of oxygen (baseline). The physical exam revealed a female with no signs of distress who was well-hydrated with a moist oral pharynx with no suggestive signs of exudate or masses. Her neck revealed an apparent anterior neck hematoma with significant ecchymosis radiating to the mediastinal area. The neck was supple with no palpable thyromegaly or cervical and supraclavicular lymphadenopathy. On auscultation there was no evidence of 


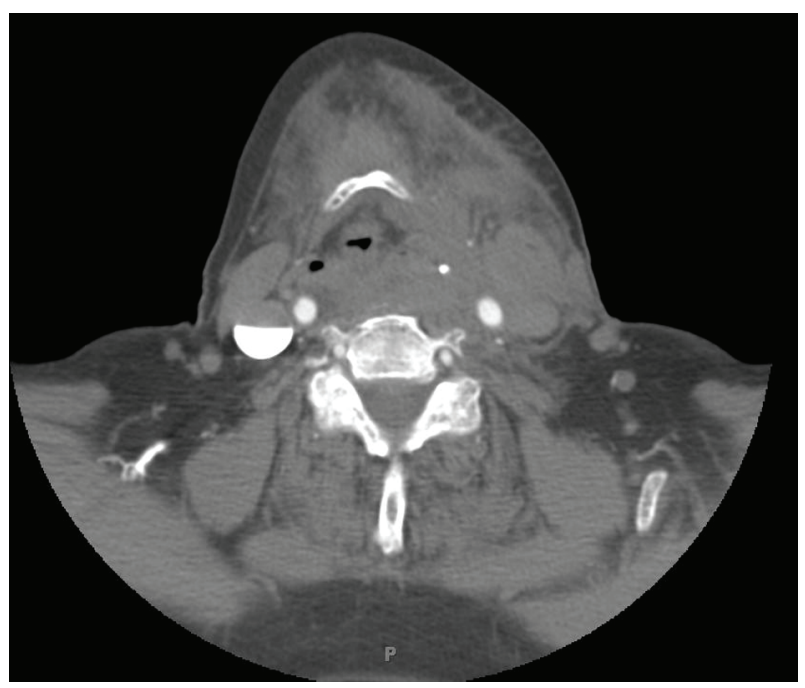

Figure 1. CT angiogram of the neck revealing no evidence of contrast extravasation to suggest an active bleed. There is infiltration of the subcutaneous fat from the chin to lower neck consistent with a history of superficial hematoma.

bruits with a regular irregular heart sounds and mild bibasilar prolonged expiratory wheezing.

Her initial laboratory workup showed hemoglobin $12.6 \mathrm{~g} /$ $\mathrm{dL}$, hematocrit $40.0 \%$, white blood count $6,400 / \mu \mathrm{L}$ and platelets of $180,000 / \mu \mathrm{L}$. Her coagulation profile revealed a protime of $16.5 \mathrm{~s}$, activated partial thromboplastin time of $37.4 \mathrm{~s}$ and INR of 1.4. Her albumin was $3.3 \mathrm{~g} / \mathrm{dL}$, her electrolytes and kidney function were within normal limits including calcium at $9.7 \mathrm{mg} / \mathrm{dL}$ (normal range: $8.4-10.5 \mathrm{mg} / \mathrm{dL}$ ) with a corrected level of $10.3 \mathrm{mg} / \mathrm{dL}$.

The concern in the ED was a dissection/aneurysm of the neck vasculature. A CT angiogram of the neck revealed infiltration of the subcutaneous fat from the chin to the lower neck suggesting a hematoma with no evidence of vascular compromise (Fig. 1). An ultrasound of neck was also performed; however, the view was obscured by the hematoma. The patient was admitted for observation for possible airway compromise and her anticoagulation therapy was temporarily discontinued. On the medical floor, the patient went into respiratory distress that required intubation. There was clinical suspicion for an expanding hematoma that would require surgical evacuation, thus, an emergent CT of the soft tissue neck was performed that would reveal similar findings as on initial CT. The patient was transferred to intensive care unit (ICU) for further evaluation.

In the ICU the patient's neck exam revealed an expanding area of ecchymosis, but her hematoma remained stable. On routine laboratory observation in the ICU the patient's calcium level was noticed to be increasing to a corrected level of 12.9 $\mathrm{mg} / \mathrm{dL}$ (Fig. 2). The patient's medications were thoroughly reviewed, with no evidence of supplemental calcium or vitamin D. The parathyroid hormone (PTH) level was checked which was $134 \mathrm{pg} / \mathrm{mL}$ (upper limit of normal of $65 \mathrm{pg} / \mathrm{mL}$ ), and the phosphorus level was found to be low at $2.4 \mathrm{mg} / \mathrm{dL}(2.7 \mathrm{mg} / \mathrm{dL}$ the lower limit of normal). The patient's intravenous hydration was increased. Three days later the PTH continued to increase to a plateau of $176 \mathrm{mg} / \mathrm{dL}$ with decreasing corrected calcium of $11.3 \mathrm{mg} / \mathrm{dL}$. Two days later the PTH level returned closer within normal limits at $68 \mathrm{mg} / \mathrm{dL}$ with corrected calcium of $11.2 \mathrm{mg} / \mathrm{dL}$ (Fig. 3). The hematoma did not require evacuation, and clinically started to subside by the fifth day and allowing a successful extubation.

The patient continued to be in Afib, and it was decided that it would be most beneficial to hold anticoagulation therapy for the near future and have the patient take $81 \mathrm{mg}$ aspirin. The patient was transferred to inpatient rehabilitation. The calcium, albumin and PTH levels were repeated 3 weeks later (Fig. 2, $3)$.

\section{Discussion}

The first reported case of spontaneous neck hematoma that resulted in mortality was reported in 1934 [4]. Since then, a very few cases have been reported [5]. Ruptured parathyroid

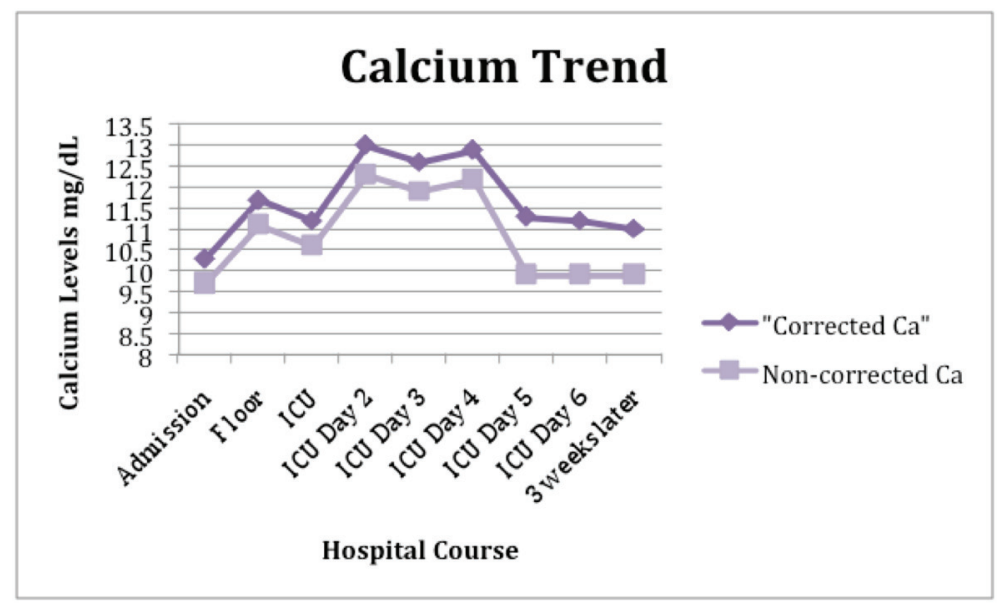

Figure 2. The corrected and non-corrected calcium levels. On ICU day 2, treatment was initiated for the hypercalcemia. On ICU day 5 the patient was successfully extubated. 


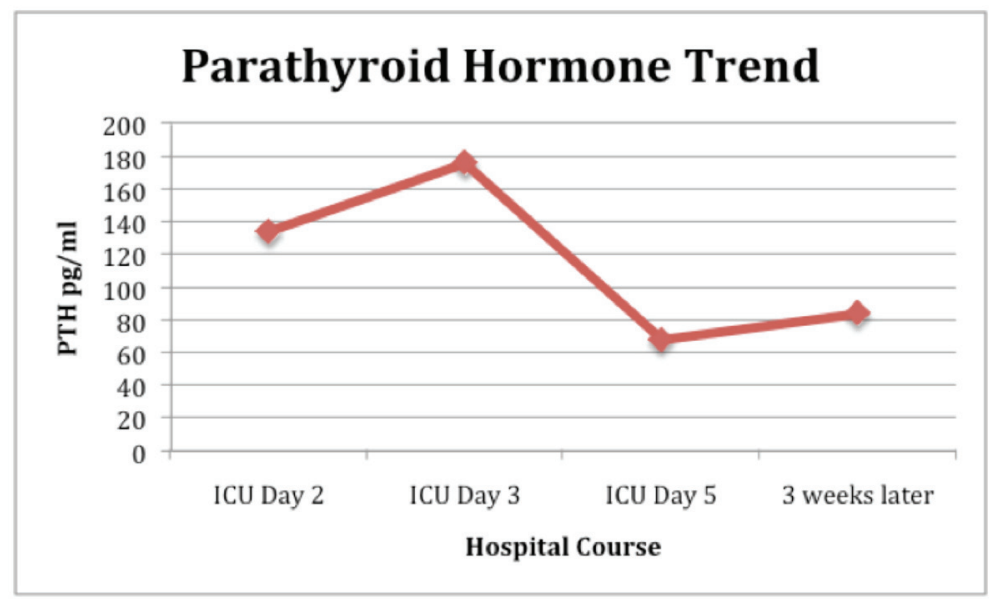

Figure 3. The PTH levels that were checked starting on ICU day 2. The level peaked on the third day and then declined within normal limits clinically correlating with decrease in anterior neck hematoma.

adenomas are difficult to diagnose. Due to intricate anatomy of parathyroid and thyroid gland, clinicians are challenged to pinpoint exact etiology of the neck hematoma. However, a clinical triad of hypercalcemia, acute neck swelling and neck or chest ecchymosis suggests a ruptured adenoma as a possible cause [6]. The exact pathophysiology is largely unknown, but the imbalance between cell growth and blood supply is believed to be a possible etiology. This imbalance would increase the propensity toward the formation of necrotic-hemorrhagic foci; this leads to bleeding [7]. In general, spontaneous neck hematomas are unusual events. The concomitant use of an anticoagulant increases the severity of neck hematoma. The expanding hematoma could jeopardize the surrounding structures such as trachea, and neurovascular bundles [2]. This could lead to devastating consequences. Patients taking anticoagulants and non-steroidal anti-inflammatory drugs are at increased risk of bleeding. When a hemorrhagic event takes place while the patient is taking warfarin, the drug could be reversed with vitamin K and fresh frozen plasma. Novel anticoagulants such as rivaroxaban are very efficacious; however, the major drawback is lack of antidotes [8]. As in our case, rivaroxaban should be held until it is eliminated from the body.

The diagnosis of ruptured parathyroid adenoma requires a high index of suspicion. The other possibilities include thyroid cysts or nodules rupture. However, the thyroid lesion hemorrhages are usually intracapsular and do not manifest as neck ecchymosis [9]. A common presentation of hyperparathyroidism due to adenoma is hypercalcemia and elevated PTH. During the hemorrhagic event from the parathyroid adenoma, calcium levels are usually elevated. Imaging studies such as CT scan and MRI are highly beneficial to reach the diagnosis. In some cases, scintigraphy can help distinguish between thyroid and parathyroid gland. Uncomplicated cases can be managed conservatively under close observation, while patients who display signs of respiratory distress and neurologic symptoms should be emergently taken for surgical evaluation. The surgical evacuation of hematoma provides immediate relief from airway compromise [9]. Furthermore, there are no established recommendations for standardized follow-up for patients with acute cervico-mediastinal hematomas [10]. As seen in our patient the calcium and PTH levels had fluctuated, but overall were trending within the normal range correlating with the resolution of the hematoma. This phenomenon could be explained by the principle of autoparathyroidectomy as a result of either intra- or extra-capsular hemorrhage; the PTH and calcium levels are corrected without surgical removal of the parathyroid adenoma [11].

\section{Conclusion}

A spontaneous rupture of parathyroid adenoma is rare and can be life-threatening. Our case highlights an important issue of novel anticoagulant and hemorrhagic complication of parathyroid adenoma. Complicated cases might require emergent surgical evaluation to avoid airway and neurovascular compromise. Since anticoagulants and antiplatelets are known to increase the likelihood of spontaneous bleeding and with many new anticoagulants/antiplatelets that have streamlined, with side-effect profile not completely revealed, it is critical to be aware that this complication may arise while on rivaroxaban therapy.

\section{Conflict of Interest}

None.

\section{References}

1. Cohen O, Yehuda M, Adi M, Lahav Y, Halperin D. Spontaneous neck hematoma in a patient with fibromuscular dysplasia: a case report and a review of the literature. Case Rep Otolaryngol. 2013;2013:352830.

2. Pazardzhikliev DD, Yovchev IP, Zhelev DD. Neck hematoma caused by spontaneous common carotid artery rupture. Laryngoscope. 2008;118(4):684-686. 
3. Bloom DC, Haegen T, Keefe MA. Anticoagulation and spontaneous retropharyngeal hematoma. J Emerg Med. 2003;24(4):389-394.

4. RC. Multiple parathyroid tumors with massive mediastinal and subcutaneous hemorrhage: a case report. Journal of American Medical Science. 1934;188:800-805.

5. Merante-Boschin I, Fassan M, Pelizzo MR, Ide EC, Rugge M. Neck emergency due to parathyroid adenoma bleeding: a case report. J Med Case Rep. 2009;3:7404.

6. Simcic KJ, McDermott MT, Crawford GJ, Marx WH, Ownbey JL, Kidd GS. Massive extracapsular hemorrhage from a parathyroid cyst. Arch Surg. 1989;124(11):13471350 .

7. Howard JE, Follis RH, Jr., Yendt ER, Connor TB. Hyperparathyroidism; case report illustrating spontaneous remission due to necrosis of adenoma, and a study of the incidence of necroses in parathyroid adenomas. J Clin Endocrinol Metab. 1953;13(8):997-1008.

8. Bauer KA. Reversal of antithrombotic agents. Am J Hematol. 2012;87(Suppl 1):S119-126.

9. Huang J, Soskos A, Murad SM, Krawisz BR, Yale SH, Urquhart AC. Spontaneous hemorrhage of a parathyroid adenoma into the mediastinum. Endocr Pract. 2012;18(4):e57-60.

10. Yoshimura N, Mukaida H, Mimura T, Iwata K, Amioka A, Hirabayashi N, Takiyama W. A Case of an Acute Cervicomediastinal Hematoma Secondary to the Spontaneous Rupture of a Parathyroid Adenoma. Ann Thorac Cardiovasc Surg. 2013.

11. Wootten CT, Orzeck EA. Spontaneous remission of primary hyperparathyroidism: a case report and meta-analysis of the literature. Head Neck. 2006;28(1):81-88. 